Supporting Information

\title{
Contact Angle Hysteresis on Smooth/Flat and Rough Surfaces.
}

\author{
Interpretation, Mechanism and Origin \\ Kock-Yee Law*
}

Research and Innovative Solutions, 27 Valewood Run, Penfield, NY 14526, USA

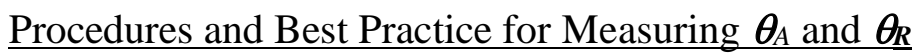

There are three methods to measure $\theta_{A}$ and $\theta_{R}$ : sessile drop expansion/contraction method, tilting plate method and the Wilhelmy plate technique. Details of the procedures have recently been reviewed. ${ }^{1}$ The pros and cons of the three methods are briefly discussed as follows. Among the three methods, Wilhelmy plate technique is most accurate, but it is less popular due to the strict requirement for sample preparation and the use of a different equipment (a tensiometer) from the other two methods. Many researchers like to use the tilting plate method with a goniometer, where they can measure sliding angle $\alpha, \theta_{A}$ and $\theta_{\boldsymbol{R}}$ at the same time. Few aware of its limitation. Figure S1 shows a schematic of a sessile droplet on an inclined surface with tilted angle $\boldsymbol{\alpha}$ and lead/trial edge angles $\boldsymbol{\theta}_{\text {max }}$ and $\boldsymbol{\theta}_{\text {min }}$. At the moment of sliding, $\boldsymbol{\alpha}$ is the sliding angle and $\boldsymbol{\theta}_{\max }=\boldsymbol{\theta}_{\mathrm{A}}$ and $\boldsymbol{\theta}_{\min }=\boldsymbol{\theta}_{\boldsymbol{R}}$. In 2008, Pierce, Carmona, and Amirfazli ${ }^{2}$ compared $\theta_{A}$ and $\theta_{\boldsymbol{R}}$ obtained from the drop expansion/contraction method with those measured from the tilting plate method on two different surfaces and the results are summarized in Table S1.

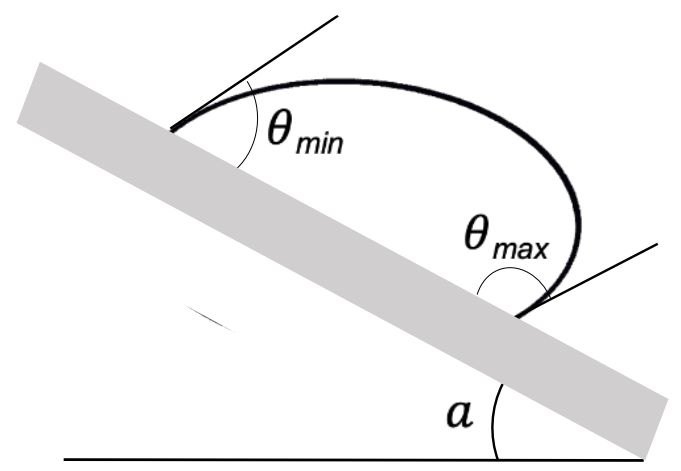

Figure S1. Schematic showing the relationship between $\theta_{\max }, \theta_{\min }$, and $\alpha$ and the forces acting on a liquid droplet on an inclined solid surface. 
Table S1. Comparison of $\boldsymbol{\theta}_{A}$ and $\boldsymbol{\theta}_{\boldsymbol{R}}$ measured from the drop expansion/contraction method to the tilting plate method (data from Ref. 2)

\begin{tabular}{ccccc}
\hline & \multicolumn{2}{c}{$\begin{array}{c}\text { Drop expansion/ } \\
\text { contraction method }\end{array}$} & & \multicolumn{2}{c}{ Tilting plate method } \\
& $\theta_{A}$ & $\theta_{\boldsymbol{R}}$ & $\theta_{A}$ & $\theta_{\boldsymbol{R}}$ \\
\hline Teflon coated Silicon & $\sim 128^{\circ}$ & $\sim 115^{\circ}$ & $\sim 124^{\circ}$ & $\sim 112^{\circ}$ \\
Alkyl ketene dimer (AKD) & $\sim 163^{\circ}$ & $<10^{\circ}$ & $\sim 163^{\circ}$ & $\sim 83^{\circ}$ \\
surface & & & & \\
\hline
\end{tabular}

The results show that, both methods produce very similar $\theta_{A}$ and $\theta_{R}$ on Teflon. Different results were obtained on AKD. $\theta_{A}$ and $\theta_{R}$ were determined to be at $163^{\circ}$ and $<10^{\circ}$ with the drop expansion/contraction method, whereas different result was obtained with the tilting plate method. $\theta_{A}$ and $\theta_{R}$ were measured at $163^{\circ}$ and $83^{\circ}$, respectively. Inspection of the receding measurement data in the original report clearly shows that the sessile droplet is in mechanical equilibrium throughout the receding measurement on Teflon, but that equilibrium was never achieved on the AKD surface. The receding angle decreases continuously as liquid is withdrawn. The large discrepancy in $\theta_{R}$ between the two methods is attributable to the strong water-AKD surface adhesion, which drags the contact line as it recedes. As a general guidance, we would not recommend anyone using the tilting plate method to determine $\theta_{A}$ and $\theta_{R}$, especially on surfaces with large hysteresis.

Microscopy and Surface Properties of Model Superhydrophobic and Superoleophobic Surfaces I

Figure S2a shows the SEM micrograph of model superhydrophobic and superoleophobic surface (I) consisting of $3 \mu \mathrm{m}$ diameter pillar array fabricated by photolithographic technique followed by surface fluorination with tridecafluoro-1,1,2,2tetrahydrooctyltrichlorosilane (FOTS). The water and hexadecane contact angle data are given in Figure S2b along with the controlled surfaces. Together with the sliding angle 
data $\left(\sim 10^{\circ}\right)$ the overall results indicate that $\mathbf{I}$ is superhydrophobic and superoleophobic. Structure-property relationship study show that the wavy sidewall structure in the pillar (see Figure S2a inset) is crucial in achieving superoleophobicity, where hexadecane is shown to pin underneath the re-entrant structure. ${ }^{3}$
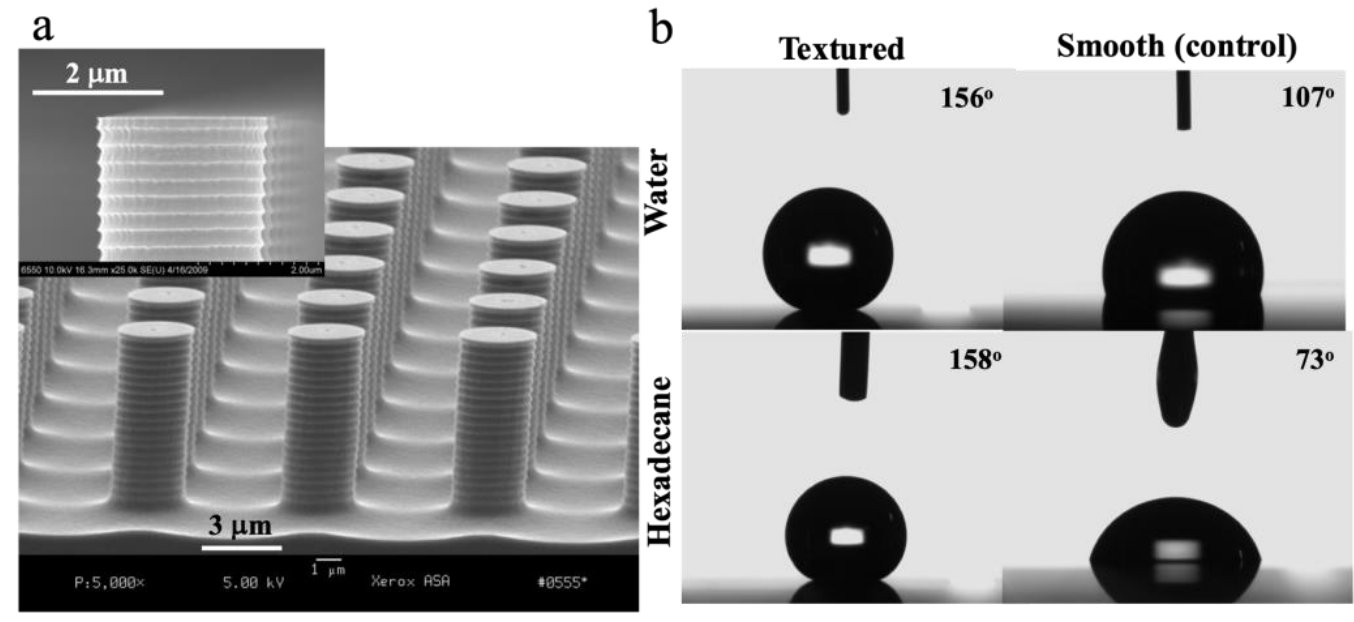

Figure S2. (a) SEM micrograph of surface I (inset: magnified micrograph of a pillar), and (b) contact angle data of I (textured) and FOTS modified Si wafer (smooth) with water and hexadecane, estimate error $< \pm 2^{\circ}$. (Reproduced with permission from Ref. 3, Copyright 2012 American Chemical Society)

\section{Brief Review of Literature on Wettable Slippery Surfaces}

Surfaces that exhibit simultaneous low contact angle and small sliding angle have been reported on-and-off in the last 90 years. For instances, Langmuir ${ }^{4}$ observed a completed roll-off of a white mineral oil droplet on a trimolecular stearate LangmuirBlodgett (LB) film on glass, which exhibited a contact angle of $\sim 55^{\circ}$. He described the unexpected observation as "de-wetting", attributable to both the tight packing of the C18 hydrocarbon chain in the LB film as well as the non-interacting nature of the $\mathrm{CH}_{3}$ end group. In 1996, Schmidt et al. ${ }^{5}$ reported the synthesis of a family of non-sticking, wettable polymers by cross-linking reactive perfluoroalkyl polymeric surfactants with poly(2isopropenyl-2-oxazoline) at different reactant ratios under various reacting and curing conditions. Contact angles $\boldsymbol{\theta}, \boldsymbol{\theta}_{\boldsymbol{A}}, \boldsymbol{\theta}_{\boldsymbol{R}}$ and sliding angles $\boldsymbol{\alpha}$ with water and hexadecane were reported. With hexadecane, four of the polymer coatings exhibit $\theta$ range between $58^{\circ}$ to $67^{\circ}$ and $\boldsymbol{\alpha}<15^{\circ}$. Recently, surfaces with even smaller $\boldsymbol{\theta}\left(\sim 30-50^{\circ}\right)$ and $\boldsymbol{\alpha}\left(<10^{\circ}\right)$ were 
reported by several research groups: the McCarthy group at University of Massachusetts, 6,7 the Hozumi group at AIST Japan, ${ }^{8-11}$ the Law group at Xerox, ${ }^{3,12,13}$ and the Liu group at Queen's. ${ }^{14,15}$ All of these surfaces are flat and smooth based on optical microscopy and AFM studies. Without exception, they are all hydrophobic with water $\boldsymbol{\theta}>90^{\circ}$. Similar to the polyurethane-SilClean coatings, they all found to be highly wettable and highly slippery with hexadecane $\boldsymbol{\theta}$ ranges between $30^{\circ}$ to $70^{\circ}$ and sliding angles less than $10^{\circ}$.

Evidence to support the chemical compatibility and molecular relaxation mechanism discussed in the text comes from data reported by Hozumi et al. in 2012 on a series of PDMS brush grafted surfaces on Si-wafer. ${ }^{8}$ The molecular weight (MW) of the PDMS brushes varies from 2,000 to 120,000 . The surface properties were studied by advancing and receding contact angle measurements. Plots of $\left(\boldsymbol{\theta}_{\boldsymbol{A}}-\boldsymbol{\theta}_{\boldsymbol{R}}\right)$ as a function of the MW of the PDMS brush with water, n-decane, n-dodecane and n-hexadecane are reproduced in Figure S3. Consistent with earlier discussion, $\left(\boldsymbol{\theta}_{\boldsymbol{A}}-\boldsymbol{\theta}_{\boldsymbol{R}}\right)$ for water is consistently larger than those with hydrocarbon solvents, attributable to the relative incompatibility between water and the PDMS brushes. The significance of the results lies in the PDMS MW effect on the polymer brush and the hydrocarbon chain length effect for the wetting liquid. According to the proposed mechanism, a rapid molecular relaxation between liquid molecules and the PDMS brush at the liquid-solid interface during advancing would lead to $\boldsymbol{\theta}_{\boldsymbol{A}}=\boldsymbol{\theta}_{\boldsymbol{R}}$. The data in Figure S3 clearly show that $\left(\boldsymbol{\theta}_{\boldsymbol{A}}-\boldsymbol{\theta}_{\boldsymbol{R}}\right)$ decreases as the MW of the PDMS brush decreases and as the chain length of the wetting liquid decreases. The results fully support the molecular relaxation mechanism. 


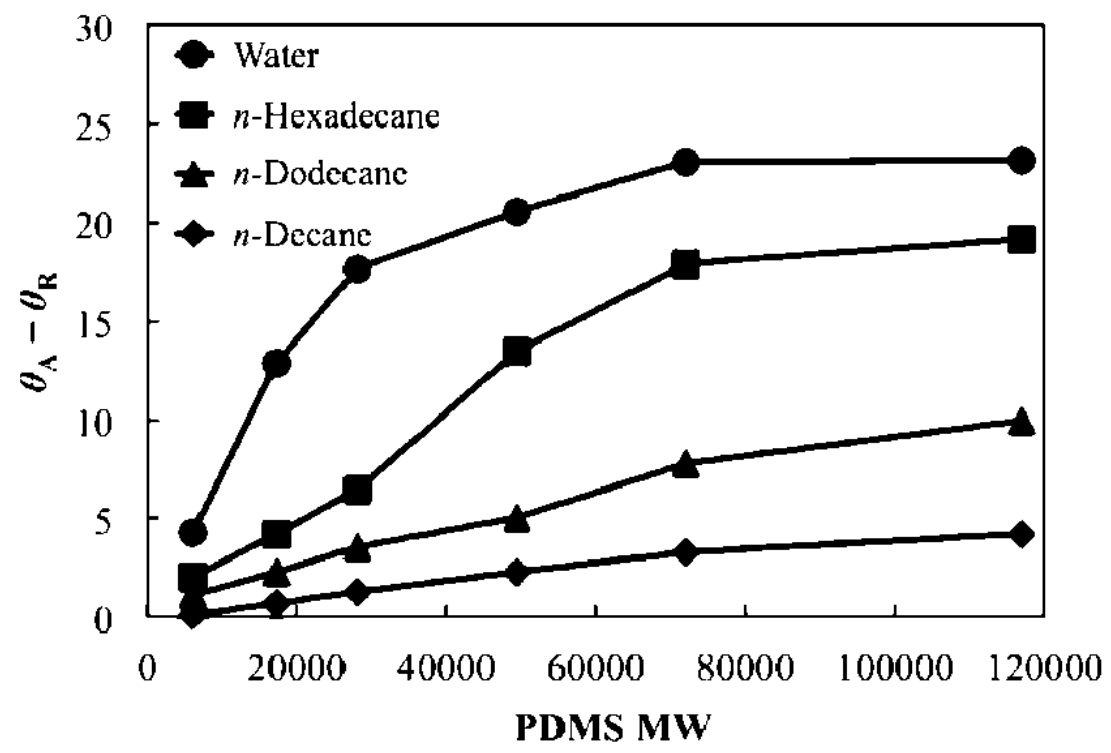

Figure S3. Change of $\left(\boldsymbol{\theta}_{\boldsymbol{A}}-\boldsymbol{\theta}_{\boldsymbol{R}}\right)$ for grafted PDMS surfaces with varying molecular weight, estimated error $< \pm 2^{\circ}$. (Reproduced from Ref. 8. Copyright American Chemical Society 2012)

\section{Effect of Water Wetting on X-linked Hydrogel Films}

In 1975, Holly and Refojo ${ }^{16}$ found that water does not fully spread on crosslinked poly(2-hydroxyethyl methacrylate) hydrogel films of water content varying from $32 \%$ to 43\%. Water $\theta_{A}$ and $\theta_{R}$ measurement showed that $\theta_{A}$ ranges from $58.1^{\circ}$ to $84.3^{\circ}$ and $\theta_{R}$ ranges from $8.2^{\circ}$ to $20.8^{\circ}$ with hysteresis varies from $40.2^{\circ}$ to $75.5^{\circ}$. The large hysteresis is indicative of stickiness. The authors attributed the larger than expected $\theta_{A}$ to the binding of water molecules in the bulk film and localization of all hydrophobic groups at the surface during film forming. A graphic representation of the chemical structure in the hydrogel film is shown in Figure S4a. After dispensing a water droplet on the hydrogel film, interaction between water molecules and the hydroxy groups as well as water in the bulk film occurs (Figure S4b). The H-bonding between the migrated water molecules and the water droplet is highlighted as blue lines. The increased interactions at the water-film interface drags the receding contact line, resulting in smaller $\theta_{\boldsymbol{R}}$ and larger hysteresis. 


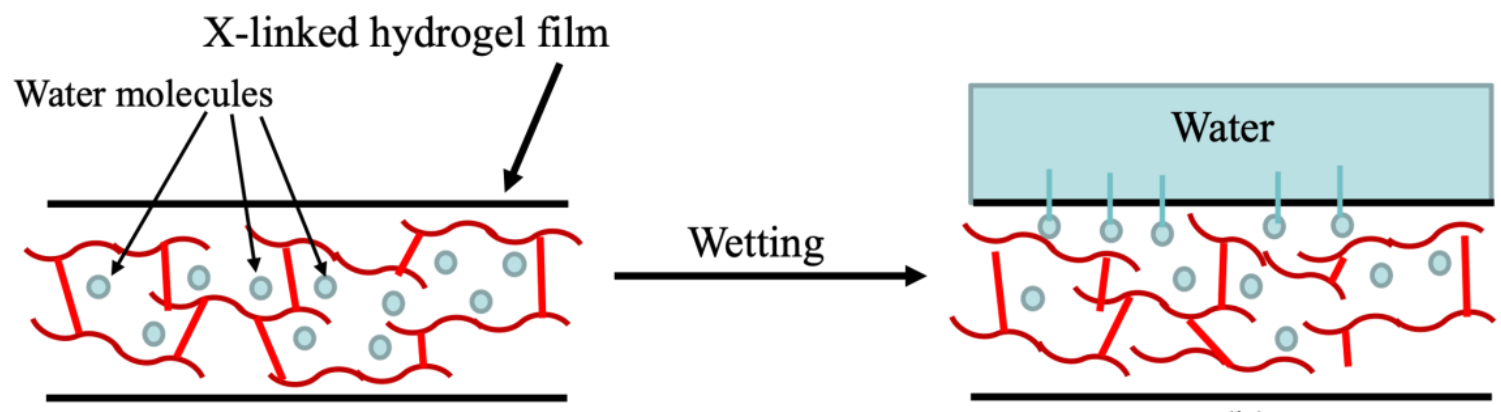

(a)

(b)

Figure S4. A schematic of the chemical structure in X-linked hydrogel film of poly(2hydroxyethyl methacrylate) before and after wetting by water.

\section{References}

1. Law, K. Y.; Zhao, H. Surface Wetting. Characterization, Contact Angle and Fundamentals, Springer, 2016, Ch 2.

2. Pierce, E.; Carmona, F. J.; Amirfazli, A. Understanding of Sliding and Contact Angle Results in Tilted Plate Experiments. Colloids and Surfaces A: Physicochem. Eng. Aspects 2008, 323, 73-82.

3. Zhao, H.; Law, K. Y.; Sambhy, V. Fabrication, Surface Properties, and Origin of Superoleophobicity for a Model Textured Surface. Langmuir 2011, 27, 5927-5935.

4. Langmuir, I. Mechanical Properties of Monomolecular Films. J. Franklin Inst. 1934, 218, 143-171.

5. Schmidt, D. L.; DeKoven, B. M.; Coburn, C. E.; Potter, G. E.; Meyers, G. F.; Fischer, D. A. Characterization of a New Family of Nonwettable Nonstick Surfaces. Langmuir 1996, 12, 518-529.

6. Chen, W.; Fadeev, A. Y.; Hsieh, M. C.; Oner, D.; Youngblood, J.; McCarthy, T. J. Ultrahydrophobic and Ultralyophobic Surfaces: Some Comments and Examples. Langmuir 1999, 15, 3395-3399.

7. Wang, L.; McCarthy, T. J. Covalently Attached Liquids: Instant Omniphobic Surfaces with Unprecedented Repellency. Angew. Chem. Int. Ed. 2016, 55, 244-248. 
8. Cheng, D. F.; Urata, C.; Masheder, B.; Hozumi, A. A Physical Approach to Specifically Improve the Mobility of Alkane Liquid Drops. J. Am. Chem. Soc. 2012, 134, 1019110199.

9. Cheng, D. F.; Masheder, B.; Urata, C.; Hozumi, A. Smooth Perfluorinated Surfaces with Different Chemical and Physical Nature: Their Unusual Dynamic Dewetting Behavior toward Polar and Non-polar Liquids. Langmuir 2013, 29, 11322-11329.

10. Urata, C.; Masheder, B.; Cheng, D. F.; Miranda, D. F.; Dunderdale, G. J.; Miyamae, T.; Hozumi, A. Why Can Organic Liquids Move Easily on Smooth Alky-Terminated Surfaces? Langmuir 2014, 30, 4049-4255.

11. Urata, C.; Cheng, D. F.; Masheder, B.; Hozumi, A. Smooth, Transparent and NonPerfluorinated Surfaces Exhibiting Unusual Contact Angle Behavior towards Organic Liquids. RSC Advances 2012, 2, 9805-9808.

12. Kovacs, G. J.; Law, K. Y.; Zhao, H.; Sambhy, V. Coating for an Ink Jet Printhead Front Face. 2012, U.S. Patent 8,226,207.

13. Sambhy, V.; Law, K. Y.; Zhao, H.; Gulvin, P. M.; Casella, J. M. Improved Process for Thermally Stable Oleophobic Low Adhesion Coating for Inkjet Printhead Front Face. 2015, U.S. Patent 9,073,323.

14. Hu, H.; Liu, G.; Wang, J. Clear and Durable Epoxy Coatings that Exhibit Dynamic Omniphobicity. Adv. Mater. Interface, 2016, 1600001.

15. Rabnawaz, M.; Liu, G.; Hu, H. Graft-Copolymer Based Approach to Clear, Durable, and Anti-Smudge Polyurethane Coatings. Angew. Chem. Int. Ed. 2015, 54, 6516-6520.

16. Holly, F. J.; Refojo, M. F. Wettability of Hydrogels 1. Poly(2-hydroxyethyl methracrylate). J. Biomed. Mater. Res. 1975, 9, 315-326. 